\title{
Intelligent Data Rate Control in Cognitive Mobile Heterogeneous Networks
}

\author{
Jeich Mar \\ Department of Communications Engineering \\ Yuan-Ze University \\ 135 Yuan-Tung Road, Jungli, \\ Taoyuan 320, Taiwan, R.O.C \\ eejmar@saturn.yzu.edu.tw
}

\author{
Hsiao-Chen Nien \\ Department of Communications Engineering \\ Yuan-Ze University \\ 135 Yuan-Tung Road, Jungli, \\ Taoyuan 320, Taiwan, R.O.C \\ s940701@mail.yzu.edu.tw
}

\begin{abstract}
An adaptive rate controller (ARC) based on adaptive neural fuzzy inference system (ANFIS) is designed to autonomously adjust the data rate of a mobile heterogeneous network to adapt the changing traffic load and the user speed for multimedia call services. Through simulations, it is demonstrated that the ARC is able to maintain new call blocking probability and handoff failure probability of the mobile heterogeneous network below a prescribed low level over different user speeds and new call origination rates while maximizing the average throughput. It is also shown that the ARC can make mobile heterogeneous network to accommodate more traffic load.
\end{abstract}

Keywords- complete sharing (CS) protocol, hierarchical Mobile WiMAX and DSRC networks, cognitive mobile heteregeneous networks, adaptive rate controller (ARC)

\section{INTRODUCTION}

The tremendous growth of the wireless/mobile user population, coupled with bandwidth requirements of multimedia applications, requires efficient management in the view of quality of service $(\mathrm{QoS})$ provisioning. The $2.5 \mathrm{G}$ and $3 \mathrm{G}$ cellular systems employ radio resource management (RRM) strategies that allow them not only to assign variable bandwidth to incoming voice or data calls but also dynamically vary the bandwidth of ongoing calls. These RRM strategies are known as flexible (adaptive) resource allocation (FRA) strategies and their ultimate end is to find a tradeoff between the existing capacity and QoS. Various FRA schemes have been proposed and investigated [1]. In [2], a resource management strategy for heterogeneous adaptive-rate traffic is presented. In [3], a novel, rate-based, borrowing scheme for QoS provisioning in high-speed cellular networks carrying multimedia traffic is presented. With the QoS provisioning and the problem of scarce bandwidth resource, keeping the new call blocking probability and handoff failure probability lower than predefined threshold while at the same time maximizing the network throughput is one of the most challenging tasks for flexible (adaptive) bandwidth allocation strategies [4][5].

In this paper, we propose an adaptive rate controller (ARC) based on adaptive neural fuzzy inference system (ANFIS) [6] for multimedia mobile heterogeneous network. The ARC chooses new call blocking probability, handoff failure probability, new call origination rate and user speed as linguistic variables to adaptively allocate bandwidth to every new and handoff multimedia call. The objective optimization of the proposed ARC is able to control new call blocking probability and handoff failure probability below a target threshold while maximizing average throughput subject to the constraint of the limited bandwidth of the mobile heterogeneous network. The ANFIS control rule is designed through simulations of the traffic performance for the mobile heterogeneous network, where the hierarchical Mobile WiMAX and DSRC networks are considered as an example. For simplicity, we employ the protocol allowing all kinds of traffic services to share all the bandwidth resource of a cell with a first come first service rule.

\section{PRINCIPLES}

The system architecture of the ARC based on ANFIS is shown in Fig. 1, which is divided as CS-ANFIS-ARC mode and $\mathrm{CS}$ mode. The total number of basic bandwidth units (BBUs), C, are assigned for each system. One BBU equals to the data rate of $64 \mathrm{kbps}$ (VoIP applications require $64 \mathrm{kbps}$ ) [7]. The maximum number of users is assumed to be 40 , which should be less than the number of data sub-carriers of the Mobile WiMAX/DSRC OFDM systems. The cell radii of the Mobile WiMAX and DSRC systems are assumed to be $2 \mathrm{~km}$ and $100 \mathrm{~m}$, respectively. There are four type of new call and handoff call services, including UGS (Unsolicited Grant Service), BE (Best Effort), nrtPS (non-real-time Polling Service), rtPS (real-time Polling Service). UGS is designed for voice call services requiring a fixed packet interval and fixed packet size such as VoIP application.

The ARC based on ANFIS consists of a CS frequency channel allocation rule and an ARC. When the network traffic is under-loaded, the ARC enables the CS mode to allocate maximum data rate for the software-defined radio (SDR) radio transceiver to set a channel coding rate and a modulation type, but in the time during which over-loaded network traffic occurs the CS-ANFIS-ARC mode is enabled to receive the feedback information from the network controllers of the Mobile WiMAX/DSRC system to reduce its allocating data rate. When every new or handoff call generates in a given cell, the ARC based on ANFIS allocates a proper data rate for multimedia service calls based on the feedback internal network QoS state information and external environmental conditions including user speed and traffic loading. The ARC based on ANFIS will control new call blocking probability and handoff failure 
probability below a target threshold while maximizing the throughput. The target threshold of new call blocking probability and handoff probability is set as 0.05 .

\section{A. Mobility model for new call and handoff call}

First, in each cell, the new call arrival rate $\lambda$ (calls $/ \mathrm{sec}$ ) follows Poisson process. The bandwidth holding time is defined as the time duration between the instant that the bandwidth is occupied by a new (or handoff) call and the instant it is released by either call completion or call handoff again. The bandwidth holding time $T_{H n, i, k}$ for a new call in system $i$ and type $k$ call service is the minimum of the unencumbered call holding time [8] $T_{k}$ for type $k$ call service in system $i$ and the cell residence time $T_{n, i}$ for a new call in system $i$. The cell residence time $T_{n, i}$ is defined as the duration a call resides in system $i$. We assume the call holding time for a new call has a negative exponential distribution. The bandwidth holding time $T_{H h, i, k}$ for a handoff call in system $i$ and type $k$ call service is either the remaining call holding time $T_{h k}$ or the cell residence time $T_{h, i}$ for a handoff call in system $i$, whichever is less. The random variable $T_{h k}$ is given by

$$
T_{h k}=T_{k}-T_{n, i}-(c-1) \times T_{h}
$$

where $c$ is the number of handoff for a given call. The random variables for $T_{H n, i, k}$ and $T_{H h, i, k}$ are given by

$$
\begin{aligned}
& T_{H n, i, k}=\min \left(T_{k}, T_{n, i}\right) \\
& T_{H h, i, k}=\min \left(T_{h k}, T_{h, i}\right)
\end{aligned}
$$

The distance between the point where a new call is originated and the point on cell boundary where it exits from cell is $Z_{i}$. Then the cell residence time of for a new call $T_{n}$ is expressed as $T_{n, i}=Z_{i} / V_{i}$. This is a function of system parameters such as cell size, speed and direction of movement by a call. Then the probability density functions (pdfs) of the cell residence time $T_{n}$ for a new call and a handoff call are given in[8], where $V_{\max }$ and $V_{\min }$ are the maximum and minimum speed of a call in a cell, respectively. The speed of a call in the given cell is uniformly distributed in $\left[V_{\min }, V_{\max }\right]$. Its mean speed is $\left(V_{\min }+\right.$ $\left.V_{\max }\right) / . r_{i}$ is the cell radius of the system $i$.

\section{B. ANFIS Architecture}

The ANFIS rule employs the adaptive network architecture to represent the fuzzy inference system, which can be applied to a wide range of areas, such nonlinear function modeling, time series prediction, and fuzzy controller design. Here we use the ANFIS rule to control the new call blocking probability and handoff failure probability of the mobile heterogeneous network below a target threshold while maximizing the throughput. The ARC based on ANFIS is implemented by a five-layer ANFIS rule [6]. Four input linguistic variables are chosen for determining the data rate to achieve objective optimization of maximum average throughput. Each variable is divided into two fuzzy terms in order to reduce the computing load. The node functions of each layer are described as below:

Layer 1: Every node $i$ in this layer is an adaptive node with Gaussian membership functions (MF)

$$
\begin{aligned}
& A_{k}\left(P_{N}(n)\right)=\frac{1}{1+\left|\frac{P_{N}(n)-c_{1, j}}{a_{1, j}}\right|^{2 b_{k, j}}}, B_{k}\left(P_{H}(n)\right)=\frac{1}{1+\left|\frac{P_{H}(n)-c_{k, j}}{a_{k, j}}\right|{ }^{2 b_{k, j}}}, \\
& C_{k}(N R)=\frac{1}{1+\left|\frac{N R-c_{k, j}}{a_{k, j}}\right|^{2 b_{k, j}}}, D_{i}(V)=\frac{1}{1+\left|\frac{V-c_{k, j}}{a_{k, j}}\right|^{2 b_{k, j}}}
\end{aligned}
$$

$O_{1, k}=A_{k}\left(P_{N}(n)\right), O_{2, k}=B_{k}\left(P_{H}(n)\right), O_{3, k}=C_{k}(N R), O_{4, k}=D_{k}(V) ;$ for $k=1,2$

Layer 2: Every node $i$ in this layer is a fixed node labeled $\Pi$ The output of node $i$, denoting by $O_{2, i}$, is the minimum value of all the incoming signals for the $i$-th rule. It is given by

$$
w_{i}=O_{2, i}=\left\{\begin{array}{c}
\min \left(A_{1}\left(P_{N}(n)\right), B_{1}\left(P_{H}(n)\right), C_{1}(N R), D_{1}(V)\right) \\
\vdots \\
\min \left(A_{2}\left(P_{N}(n)\right), B_{2}\left(P_{H}(n)\right), C_{2}(N R), D_{2}(V)\right)
\end{array}, \text { for } i=1 \sim 16\right.
$$

Layer 3: Every node $i$ in this layer is a fixed node labeled $\mathrm{N}$. The $i$-th node calculates the ratio of the $i$-th rule's firing strength to the sum of all rules' firing strength. The output of node $i$, denoted by $O_{3, i}$, is called normalized firing strength and calculated as

$$
O_{3, i}=\overline{w_{i}}=\frac{w_{i}}{\sum_{j=1}^{16} w_{j}}, \text { for } i=1 \sim 16
$$

Layer 4: Every node $i$ in this layer is an adaptive node with node function

$$
O_{4, i}=\overline{w_{i}} \times f_{i}=\overline{w_{i}} \times\left(\alpha_{i} P_{N}(n)+\beta_{i} P_{H}(n)+\gamma_{i} N R(n)+\omega_{i} x_{4}+V(n)\right) ; \text { for } i=1 \sim 16(6)
$$

, where $O_{4, i}$ is the output, $f_{i}$ is a crisp output in the consequence, and the are the consequent parameter set of node $i$. The 16 fuzzy inference rules of $f_{i}$ corresponding to Fig. 2 are constructed.

Layer 5: The single node in this layer is a fixed node labeled $\Sigma$, which computes the overall output $O_{5}$ as the summation of all incoming signals

$$
Z=O_{5, i}=\sum_{i=1}^{16} O_{4, i}
$$

The ARC based on ANFIS chooses new call blocking probabilities $P_{N}(t)$, handoff failure probabilities $P_{H}(t)$, new call origination rate $N R$ and mobile user speed $V$ as linguistic variables. The new call blocking probabilities and handoff failure probabilities are defined as

$$
\begin{gathered}
P_{N}(t)=\frac{\sum_{t=0}^{t_{c}} N_{N B}(t)}{\sum_{t=0}^{t_{c}} N_{N}(t)}, 0 \leq t \leq T \\
P_{H}(t)=\frac{\sum_{t=0}^{t_{c}} H_{F}(t)}{\sum_{t=0}^{t_{c}} N_{H}(t)}, 0 \leq t \leq T
\end{gathered}
$$

, where $N_{N B}(t)$ is the number of blocked new calls at time $t$; $N_{N}(t)$ is the number of new calls at time $t ; t_{c}$ is the current time and $T$ is the total simulation time. New call origination rate follows Poisson process and mobile user's speed $V$ decides the user's cell residence time as discussed before. The average throughput of Mobile WiMAX and DSRC systems is defined 


$$
R_{T}=\frac{64 \times\left(\sum_{j=1}^{N} T_{H x, i, k, j} Z_{j}\right)}{T}(\mathrm{kbps} / \mathrm{sec})
$$

, where $N$ is the total number of successful completion calls served in a given cell within the duration of $T$ sec. $T_{H x, i, k, j}$ is the bandwidth holding time either for new call or handoff call and $Z_{j}$ is the assigned number of BBUs for the $j_{t h}$ new generation call or handoff call. In the preset total simulation time $T$, the throughput $R_{T}$ can be calculated by (10).

The ARC must be trained to achieve the goal of control new call blocking probability and handoff failure probability lower than 0.05 while maximizing system throughput. The training scenario of the ARC based on ANFIS is summarized as follows:

Four types of data rates, as shown in TABLE I, are chosen for the ARC to control the modulation type and channel coding rate of the transceiver in PHY-layer. The total number of BBUs, $C$, in each cell are assumed to be 40 . The arrival process for new-call requests is Poisson with mean rate $\lambda$ (calls/s/cell), which is uniform in all a cell. The range of offered load was varied from 0.01 to 0.1 and the interval between two call arrivals is 0.01 . Five cases of user speeds including $20 \mathrm{~km} / \mathrm{h}$, $40 \mathrm{~km} / \mathrm{h}, 60 \mathrm{~km} / \mathrm{h}, 80 \mathrm{~km} / \mathrm{h}$ and $100 \mathrm{~km} / \mathrm{h}$ are considered. The call holding time are exponentially distributed with different means for different multimedia traffic types. The mean for UGS is $120 \mathrm{~s}$ and the mean for BE $、$ nrtPS and rtPS is 300 s.

Restricting new call blocking probability and handoff failure probability to lower than 0.05 , figures 2 (a) to (e) show the average throughput verse new call origination rate for 4 type BBUs and five kind user speeds. When new call arrival rate is low, user can be provided with the maximum bandwidth, i.e., type 1, and achieve the maximum average throughput. When new call arrival is high, the bandwidth allocated to user must be reduced to control new call blocking and handoff failure probability lower than predefined threshold 0.05 . The proper type of allocated bandwidth that control new call blocking and handoff failure probability lower than predefined threshold 0.05 while achieving maximum system throughput is chosen as training data for ARC to construct fuzzy inference rule and train the ANFIS architecture.

After constructing the fuzzy control rules, the premise parameters of four MFs and the consequent parameters are trained using a hybrid learning algorithm which is a two-phase learning scheme. In phase I, a self-organized learning scheme called grant descent method is used to construct the inference rules and to locate the initial MFs in layer 1; in phase II, a reinforcement learning scheme called least square estimator (LSE) is used to optimally adjust $f_{i}$ in the layer 4 for approximating the ARC output to the desired output in ANFIS control rules.

\section{SimUlationS}

To compare the performance of the proposed CS-ANFISARC mode and CS mode, a two-contiguous-cell mobile hierarchical network model with the same parameter settings was constructed in AWSIM, which is a discrete-event-driven simulator [9]. Based on the analysis of the mobility model for new call and handoff call, a number of call generators generated Poisson arrivals of new call and handoff call requests from four type service classes as described before. Figure 3 shows the handoff rates for a new call and a handoff call $(c=1)$, which illustrates that the handoff rates increase with the user speed because the cell residence time of the calls is shorter when the MS speed is faster. The handoff rate for new call is higher than that for handoff call because the call duration of handoff call is shorter than that of handoff call. Because the cell size of the Mobile WiMAX is larger than that of the DSRC, the handoff rate of the Mobile WiMAX is lower than that of DSRC. The calculated handoff rate is applied for the following simulations.

Through simulations, both the new call blocking probability and the handoff failure probability of the DSRC system are approximately equal to zero due to there are no calls in the Mobile WiMAX system handoffs or overflows to the overlaid DSRC system and the new calls generated in the DSRC system will handoff to the Mobile WiMAX system in a very short time. Figure 4 shows that the DSRC system has the same average throughput for both the CS mode and CS-ANFISARC mode because both modes can always provide the maximum data rate for the users. Figure 5(a) and 5(b) show the new call blocking probability and handoff failure probability of the Mobile WiMAX system for CS mode and CS-ANFIS-ARC mode in terms of five different user speeds. Under CS mode, as the call origination rate is increased, so are the new call blocking probability and handoff failure probability. It can be seen that the average throughput increases with new call origination rate, but both the new call blocking probability and handoff failure probability exceed 0.05 when the new call origination rate is greater than 0.07 . The high traffic load will result in the unacceptable QoS for the users of multimedia call services. Under the CS-ANFISARC mode, both the new call blocking probability and handoff failure probability are remained to lower than 0.05 when the call origination rate exceeds 0.07 . Figure 5(c) and 5(d) show the average throughputs for CS mode and CSANFIS-ARC mode, respectively, in terms of five different user speeds. Compared with CS mode, CS-ANFIS-ARC mode is able to maintain the average throughput when traffic loading is increasing.

\section{CONCLUSION}

In this paper, the ARC based on ANFIS is proposed for a cognitive mobile heterogeneous network. The simulation results indicate that the ARC based on ANFIS is able to autonomously adjust the data rates to adapt the changing user speed and new call origination rate. It is shown that the cognitive mobile heterogeneous networks with the proposed CS-ANFIS-ARC mode could increase the new call origination rate compared with the CS mode.

\section{ACKNOWLEDGMENT}

This work is partially supported by research grants from National Science Council, R.O.C. (NSC 98-2221-E-155 -074).

\section{REFERENCES}


[1] M. Mirhakkak, N. Schult, D. Thomson, "Dynamic bandwidth management and adaptive applications for a variable bandwidth wireless environment," Selected Areas in Communications, IEEE Journal on vol.19, no.10, pp.1984-1997, Oct 2001

[2] G. Schembra, "A Resource Management Strategy for Multimedia Adaptive-Rate Traffic in a Wireless Network with TDMA Access," IEEE Trans. Wireless Comm., vol. 4, no. 1, pp. 65-78, Jan. 2005.

[3] M. Ei-Kadi, S. Olariu, H. Abdel-Wahab, "Rate-based borrowing scheme for QoS provisioning in multimedia wireless networks," Parallel and Distributed Systems, IEEE Transactions on , vol.13, no.2, pp.156-166, Feb 2002.

[4] M. Naghshineh, M.Willebeek-LeMair, "End to end QoS provisioning multimedia wireless/mobile networks using an adaptive framework," Communications Magazine, IEEE, vol.35, no.11, pp.72-81, Nov 1997.

[5] G. Schembra, "A resource management strategy for multimedia adaptive-rate traffic in a wireless network with TDMA access," Wireless
Communications, IEEE Transactions on, vol.4, no.1, pp. 65- 78, Jan. 2005.

[6] Jyh-Shing Roger Jang, “ANFIS: Adaptive-Network-Based Fuzzy Inference System”, IEEE TRANSACTIONS ON SYSTEMS, MAN, AND CYBERNETICS, VOL. 23, NO. 3, MAY/JUNE 1993.

[7] IEEE standard for local and metropolitan area networks. Part 16: Air interface for Fixed and Mobile Broadband Wireless Access Systems. Amendment 2: Physical and Medium Access Control Layers for Combined Fixed and Mobile Operation in Licensed Bands and Corrigendum 1, IEEE Std. 802.16e, 2005.

[8] S. S. Rappaport, "Blocking, hand-off and traffic performance for cellular communication systems with mixed platforms," IEE Proceedings, part I Communications, Speech and Vision, vol. 45, pp. 389-401, Oct. 1993.

[9] P. O'Reilly, Simulation with Visual SLAM and AweSim, New York: Wiley, 1999.

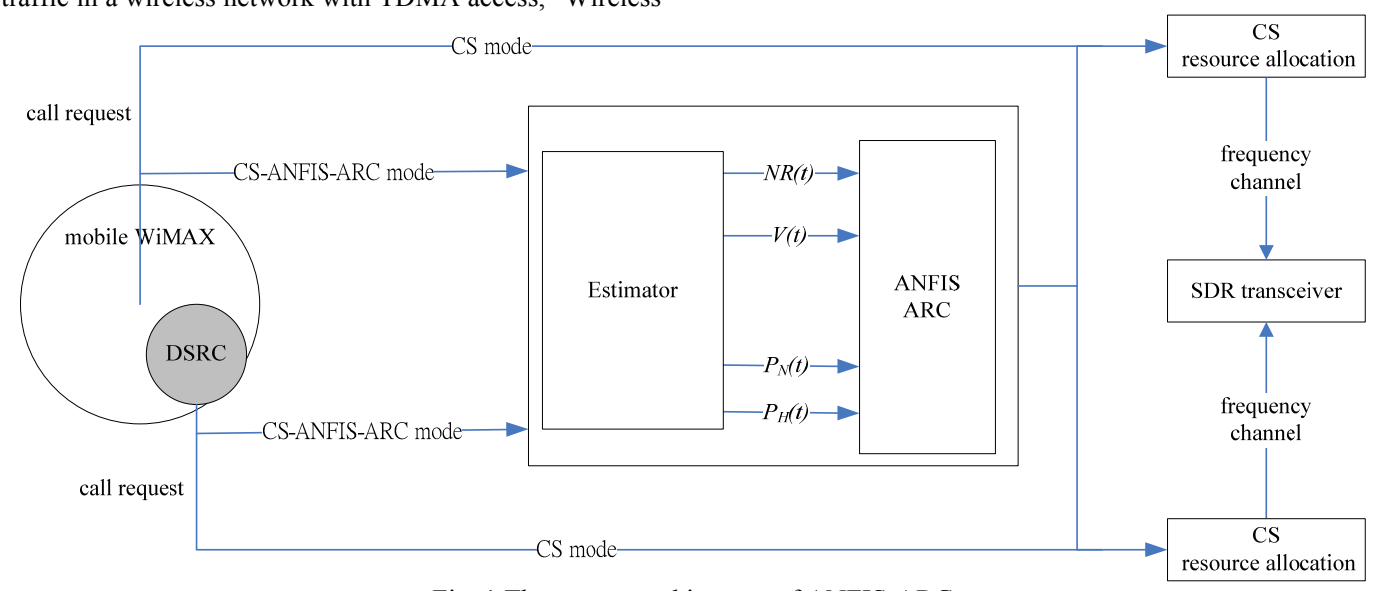

Fig. 1 The system architecture of ANFIS-ARC

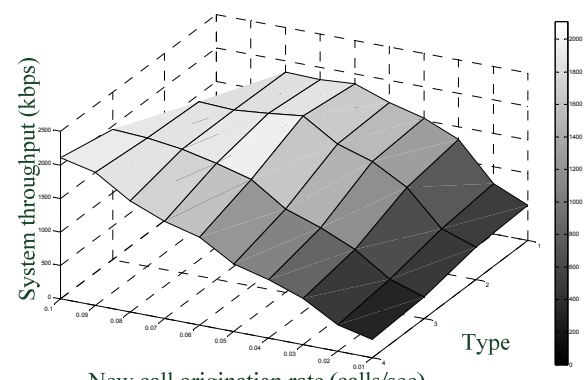

(a)

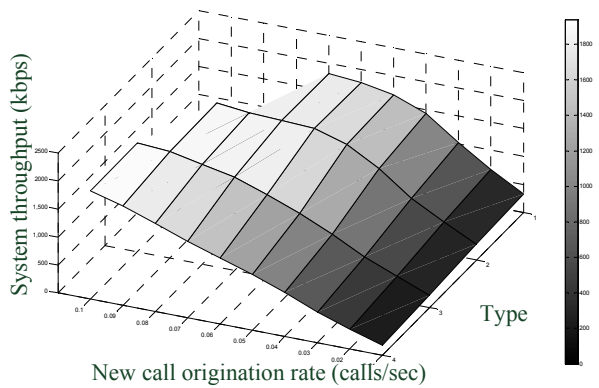

(c)

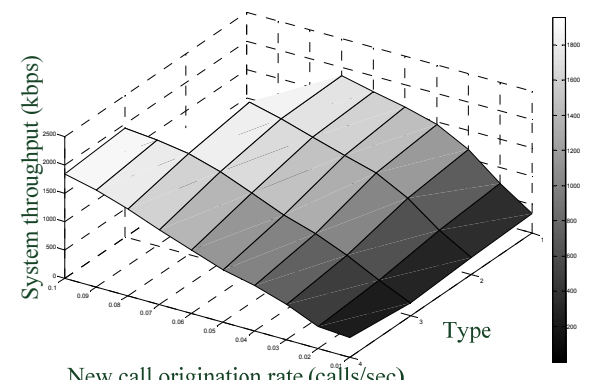

(b)

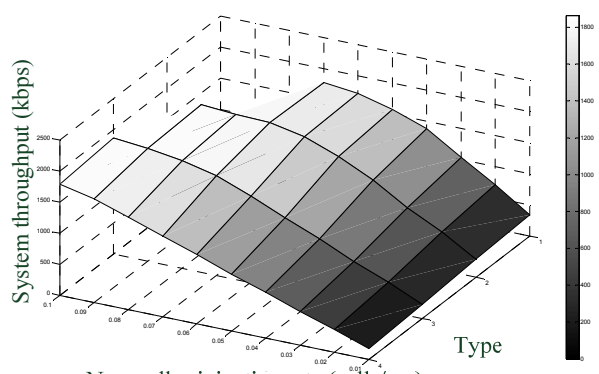

New call origination rate (calls/sec)

(d) 


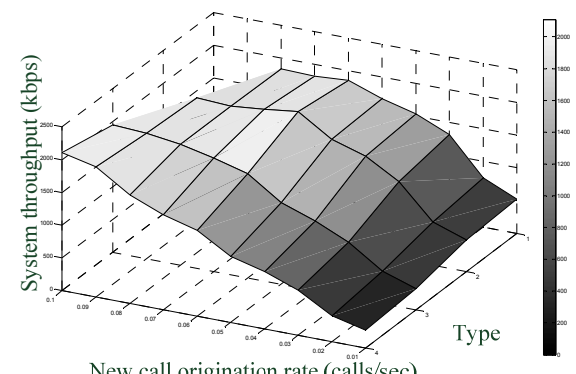

(e)

Fig.2 Mobile WiMAX training data of the ANFIS-ARC for different user speeds (a) $20 \mathrm{~km} / \mathrm{h}$, (b) $40 \mathrm{~km} / \mathrm{h}$, (c) $60 \mathrm{~km} / \mathrm{h}$, (d) $80 \mathrm{~km} / \mathrm{h}$ and (e) $100 \mathrm{~km} / \mathrm{h}$

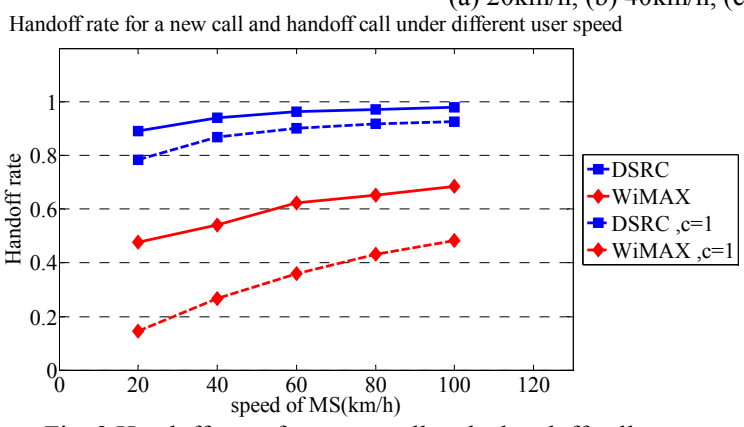

Fig. 3 Handoff rates for a new call and a handoff call

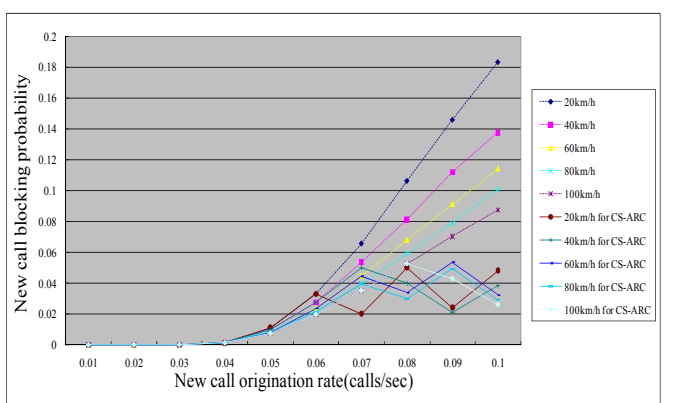

(a)

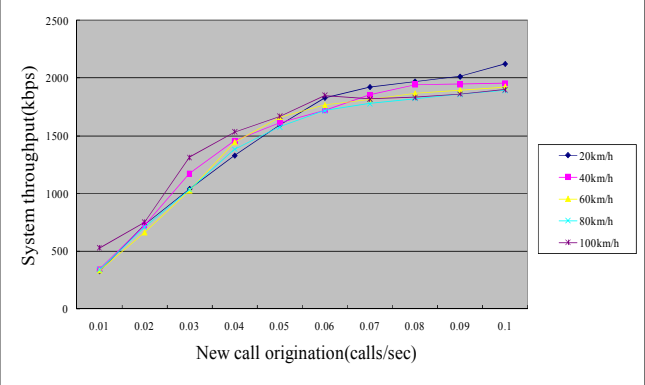

(c)

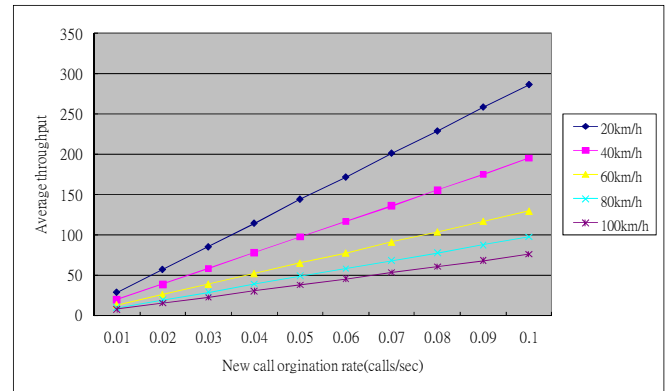

Fig. 4 Average throughput for CS and CS-ANFIS-ARC modes in DSRC system

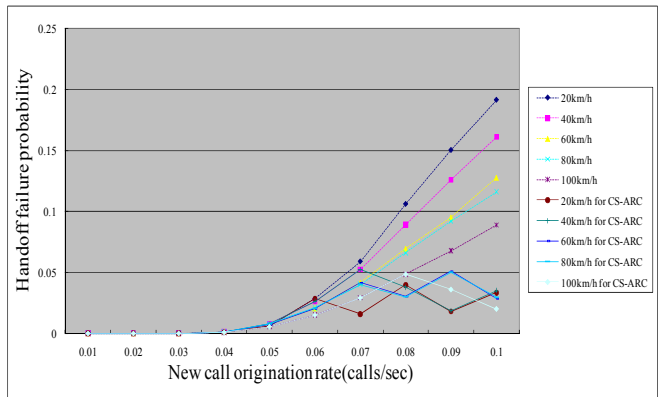

(b)

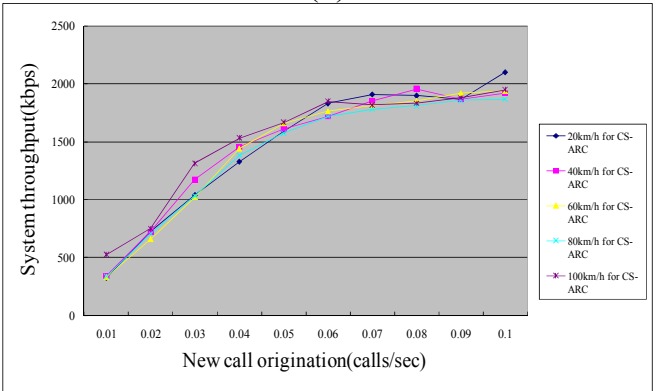

(d)

Fig. 5(a) New call blocking probability for CS mode and CS-ANFIS-ARC mode, (b) handoff failure probability for CS mode and CS-ANFIS-ARC mode (c) average throughput for CS mode (d) average throughput for CS-ANFIS-ARC mode in Mobile WiMAX

TABLE I

EQUIVALENT BBUS FOR DIFFERENT DATA RATES

\begin{tabular}{cccccc}
\multicolumn{7}{c}{ EQUIVALENT BBUS FOR DIFFERENT DATA RATES } \\
\hline \hline & Data rate & UGS & BE & nrtPS & rtPS \\
\hline Type 1 & $64 \mathrm{kbps}$ & 1BBUs & 1BBUs & 1BBUs & 1BBUs \\
Type 2 & $128 \mathrm{kbps}$ & 2BBUs & 2BBUs & 2BBUs & 2BBUs \\
Type 3 & $384 \mathrm{kbps}$ & 6BBUs & 6BBUs & 6BBUs & 6BBUs \\
Type 4 & $512 \mathrm{kbps}$ & 8BBUs & 8BBUs & 8BBUs & 8BBUs \\
\hline
\end{tabular}

